\title{
Topological Dislocations and Mixed State of Charge Density Waves
}

\author{
Masahiko Hayashi(1),(2), 目 and Hideo Yoshioka ${ }^{(3)}$ \\ (1) Superconductivity Research Laboratory, ISTEC, \\ Shinonome 1-10-13, Koto-ku, Tokyo 135, Japan \\ (2) Department of Applied Physics, Delft University of Technology, \\ Lorentzweg 1, 2628 CJ Delft, The Netherlands \\ (3) Department of Physics, Nagoya University, \\ Furo-cho, Chigusa-ku, Nagoya 464-01, Japan
}

(Received: January 24, 1996)

\begin{abstract}
We discuss the possibility of the "mixed state" in incommensurate charge density waves with three-dimensional order. It is shown that the mixed state can be created by applying an electric field perpendicular to the chains. This state consists of topological dislocations induced by the external field and is therefore similar to the mixed states of superfluids (type-II superconductor or liquid Helium II). However, the peculiar coupling of charge density waves with the electric field strongly modifies the nature of the mixed state compared to the conventional superfluids. The field and temperature dependence of the properties of the mixed state are studied, and some experimental aspects are discussed.
\end{abstract}

71.45.Lr

Typeset using REVTEX 
The charge density wave (CDW) is an ordered state in which translational symmetry is broken [1]. Topological dislocations (TD's) of incommensurate CDW with three-dimensional (3D) order have been studied by several authors with connection to the phase slip phenomena in sliding CDW's [2] [ The analogy of the TD's with the dislocations in crystals has also been pointed out [6.77. However, unnoticed up to now seem to be the similarities of the TD's with the vortices in superfluids: both are topological singularities of complex order parameter. In type-II superconductors in magnetic fields and liquid Helium II in a rotating container, the vortices create a remarkable, so-called mixed state, which generates a wide variety of intriguing phenomena [8]. In this Letter, we investigate the possibility of the corresponding mixed state in CDW's and discuss its properties.

In contrast to the vortices in type-II superconductor and liquid Helium II, which induce magnetic flux and angular momentum, respectively, the TD's in CDW's induce charge polarization. Therefore, in analyzing the electrostatic properties of the TD's, we have to treat the scalar potential carefully. We start with the microscopic model of the electron-phonon system in the presence of scalar potential, and derive the Ginzburg-Landau free energy of the ordered state. The effective free energy of the TD's is obtained by integrating out the single-valued part of the phase of the order parameter, i.e., the phasons. The TD's in the CDW's are similar to the vortices in type II superconductors or superfluids, except for the coupling to the external field. We show that the mixed state is created by an external electric field in the direction perpendicular to the chains (transverse direction), which can be understood as follows. When the system tries to screen the field, the chemical potential must be changed from chain to chain so as to induce a surface charge [9]. This modulation changes the wave number of the CDW condensate, i.e., $2 k_{F}$, thus conflicts with the $3 \mathrm{D}$ order and causes frustration. We analyze this state based on the free energy of TD's. First, we determined the lower critical field $D_{c 1}$ at which the first TD appears in the system as we increase the field strength. If we increase the field further, the density of the TD's also increases. In the strong field region, the density of TD's become so high that we can treat them as a continuum. In this limit we find that the width of the mixed state is given by the 
Thomas-Fermi screening length of the normal state. If we increase the field even further, the cores of the TD's begin to overlap, thus destroying the 3D order. The characteristic field of this phenomena, we denote by $D_{c 2}$, is estimated. We argue that these properties should be experimentally observable.

First we derive the free energy of CDW from the 1D electron-phonon system. The imaginary-time action reads (here we act in the following units $\hbar=k_{B}=1$ unless noted),

$$
\begin{aligned}
S & =\int_{0}^{\beta} d \tau\left[\int d x \psi_{\sigma}^{*}\left(\partial_{\tau}+i e \varphi-\frac{1}{2 m} \partial_{x}^{2}-\mu\right) \psi_{\sigma}\right. \\
& +\sum_{q} b_{q}^{*}(\tau)\left(\partial_{\tau}+\omega_{q}\right) b_{q}(\tau) \\
& \left.+\frac{1}{\sqrt{L}} \sum_{k, q, \sigma} g_{q} a_{k+q, \sigma}^{*}(\tau) a_{k, \sigma}(\tau)\left(b_{q}(\tau)+b_{-q}^{*}(\tau)\right)\right],
\end{aligned}
$$

where $\psi_{\sigma}=1 / \sqrt{L} \sum_{k} \mathrm{e}^{i k x} a_{k, \sigma}$ and $b_{q}$ are variables expressing the electron and phonon degrees of freedom with excitation spectra $\epsilon_{k}=k^{2} / 2 m$ and $\omega_{q}$, respectively. $L, g_{q}, \beta$ and $\mu$ are the length of the chain, the electron-phonon coupling constant, the inverse of temperature, and chemical potential, respectively. The scalar potential is expressed by $\varphi$ and the charge of an electron is given by $-e$. From the above action, the free energy of the CDW can be derived in both the cases of $T \lesssim T_{c}$ and $T \ll T_{c}$. In the former case, we utilize the expansion of the order parameter up to fourth order and the scalar potential up to second order. On the other hand, in the latter case, we use a gradient expansion of the phase of the order parameters and the scalar potential [10]. When the quasi-particle excitation gap $\Delta$ is uniform, the free energy becomes in both cases simply,

$$
\begin{aligned}
F & =\frac{e^{2}}{\pi v_{F}}\left(1-f_{s}\right) \int d x \varphi^{2}+i \frac{e}{\pi} f_{s} \int d x \theta E \\
& +\frac{v_{F}}{4 \pi} f_{s} \int d x\left(\partial_{x} \theta\right)^{2}
\end{aligned}
$$

disregarding irrelevant constants. Here $\theta$ is the phase of the order parameter, $E=-\partial_{x} \varphi$ and $f_{s}=|\Delta|^{2} \pi T \sum_{\epsilon_{n}}\left(|\Delta|^{2}+\epsilon_{n}^{2}\right)^{-3 / 2}$ is the condensate density given by $4 c_{0}|\Delta|^{2}$ for $T \lesssim T_{c}$ with $c_{0}=\beta^{2} \zeta(3,1 / 2) /(4 \pi)^{2}$, and $1-\sqrt{2 \pi \beta|\Delta|} \mathrm{e}^{-\beta|\Delta|}$ for $T \ll T_{c}$, where $\zeta(3,1 / 2)$ is the zeta function. The first term of Eq. (2) expresses the screening due to the excitation of 
quasi-particles [11]. The second term describes the acceleration of the CDW and coincides with the result obtained from the "chiral transformation" at $T \ll T_{c}$ [12, 13].

The free energy of the 3D ordered state can be obtained by introducing the rigidity due to the inter-chain coupling, as follows,

$$
\begin{aligned}
F & =\int d \mathbf{r} \frac{K}{2}\left[\left\{\partial_{x} \theta(\mathbf{r})\right\}^{2}+\gamma^{2}\left\{\partial_{y} \theta(\mathbf{r})\right\}^{2}+\gamma^{\prime 2}\left\{\partial_{z} \theta(\mathbf{r})\right\}^{2}\right] \\
& +\int d \mathbf{r}\left[i J e \varphi(\mathbf{r}) \partial_{x} \theta(\mathbf{r})+\frac{1}{8 \pi}\left\{|\nabla \varphi(\mathbf{r})|^{2}+\lambda_{0}^{-2} \varphi(\mathbf{r})^{2}\right\}+i e \varphi(\mathbf{r}) \rho^{e x t}(\mathbf{r})\right],
\end{aligned}
$$

where the electrostatic energy is also included. We assume that the chains are parallel to the $x$-axis. The anisotropy is parameterized by $\gamma=\xi_{y} / \xi_{x}$ and $\gamma^{\prime}=\xi_{z} / \xi_{x}$ with $\xi_{x}$, $\xi_{y}$ and $\xi_{z}$ being the coherence lengths in $x$-, $y$ - and $z$-direction, respectively. The screening length due to the quasi-particles, $\lambda_{0}$, is given by $\lambda_{0}^{-2}=8 N_{\perp} e^{2}\left(1-f_{s}\right) / v_{F}$. The response of the system is probed by the external charge density $\rho^{e x t}(\mathbf{r})$ which induces an external field given by $\mathbf{D}_{\mathbf{k}}^{e x t}=4 \pi e i \mathbf{k} \rho_{\mathbf{k}}^{\text {ext }} / k^{2}$ where $k^{2} \equiv|\mathbf{k}|^{2} . K=N_{\perp} v_{F} f_{s} / 2 \pi$ and $J=N_{\perp} f_{s} / \pi$ are coefficients proportional to the areal density of the chains $N_{\perp}$. Note that the size of the cores of the TD's is given by $\xi_{x}, \xi_{y}$ and $\xi_{z}$ in $x-, y$ - and $z$-direction, respectively. Although the spatial variation of the amplitude of the order parameter is neglected, the present treatment is applicable to the most CDW systems for the following reason. Since the transverse size of the cores is usually smaller than the inter-chain spacing except near $T_{c}$, the dislocations mostly sit between the chains so as to minimize the free energy, and the cores thus do not affect the order of CDW. In this Letter we focus on the intrinsic properties of a clean CDW condensate and leave the effects of inhomogeneity pinning for further work.

Based on this free energy, we first clarify the screening properties of the CDW state without TD's. In this case, only the phason modes contribute to the screening and leads the free energy:

$$
F_{\text {eff }}=\sum_{\mathbf{k}}\left[\frac{1}{8 \pi}\left(\mathbf{k}^{2}+\Lambda_{\mathbf{k}}^{-2}\right)\left|\varphi_{\mathbf{k}}\right|^{2}+i e \varphi_{\mathbf{k}} \rho_{-\mathbf{k}}^{e x t}\right]
$$

where the effective screening length $\Lambda_{\mathbf{k}}$ is given as $\Lambda_{\mathbf{k}}^{-2}=\lambda_{0}^{-2}+\left(4 \pi e^{2} J^{2} / K\right) k_{x}^{2} / k_{\gamma}^{2}$ and $k_{\gamma}^{2} \equiv k_{x}^{2}+\gamma^{2} k_{y}^{2}+\gamma^{\prime 2} k_{z}^{2}$. In the low temperature limit, $\lambda_{0}$ diverges since the quasi- 
particle excitations are exponentially suppressed by the energy gap, and then only the polarization of the condensate can contribute to the screening. In the direction along the chains (longitudinal direction: $k_{y}=k_{z}=0$ ), the phason contribution completely compensates the suppressed quasi-particle contribution. Actually, the screening length becomes $\left(\lambda_{0}^{-2}+4 \pi e^{2} J^{2} / K\right)^{-1 / 2}$, which coincides with the Thomas-Fermi length of the normal state, $\lambda_{T F} \equiv\left\{8 N_{\perp} e^{2} /\left(\hbar v_{F}\right)\right\}^{-1 / 2}$. On the other hand, in the transverse direction $\left(k_{x}=0\right)$, there is no phason contribution and, consequently, no screening for $T \ll T_{c}$. This can be attributed to the "rigidity" of CDW.

Next we examine how the TD's affect the screening properties. Here we limit our discussion to straight dislocations parallel to the $z$-axis, which reduces the problem to a two-dimensional one. The density of topological charge is then $n(\mathbf{r})=\sum_{\mu} q_{\mu} \delta^{(2)}\left(\mathbf{r}-\mathbf{r}_{\mu}\right)$, where $q_{\mu}$ and $\mathbf{r}_{\mu}$ are the topological charge, $q_{\mu}=2 \pi \times$ (integer), and the position of the $\mu^{-}$ th dislocation, respectively. The phase of the order parameter is given by the relation, $(\nabla \theta)_{\mathbf{k}}=i \mathbf{k} \theta_{\mathbf{k}}^{s}+i \mathbf{k} \times \hat{\mathbf{z}} n_{\mathbf{k}} / k^{2}$, where $\theta_{\mathbf{k}}^{s}$ expresses the phason part, $\hat{\mathbf{z}}=(0,0,1)$, and $\mathbf{k}=\left(k_{x}, k_{y}, 0\right)$. In the following we consider TD's with $q_{\mu}= \pm 2 \pi$ only. By integrating out the phason mode, the free energy of TD's per unit length becomes,

$$
\begin{aligned}
\frac{F_{\text {eff }}}{L_{z}}=\sum_{\mathbf{k}} & {\left[\frac{K \gamma^{2}}{2} \frac{1}{k_{\gamma}^{2}}\left\{n_{\mathbf{k}}-\frac{i e J}{K}\left(i k_{y} \varphi_{\mathbf{k}}\right)\right\}\left\{n_{-\mathbf{k}}-\frac{i e J}{K}\left(-i k_{y} \varphi_{-\mathbf{k}}\right)\right\}\right.} \\
& \left.+\frac{1}{8 \pi}\left(k^{2}+\Lambda_{T F}^{-2}\right)\left|\varphi_{\mathbf{k}}\right|^{2}+i e \varphi_{\mathbf{k}} \rho_{-\mathbf{k}}^{e x t}\right],
\end{aligned}
$$

where $L_{z}$ is the length of the sample in the $z$-direction. Note that in the imaginary time formulation the scalar potential has to be rotated to the imaginary axis like $\varphi_{\mathbf{k}} \rightarrow i \varphi_{\mathbf{k}}$. Therefore we define the expectation value of $\varphi_{\mathbf{k}}$ by $\left\langle\varphi_{\mathbf{k}}\right\rangle \equiv i \bar{\varphi}_{\mathbf{k}}$, where $\bar{\varphi}_{\mathbf{k}}$ is the physical value.

Eq. (5) tells us that the TD's behave like a Coulomb gas with background charge $-e J\left(k_{y} \varphi_{\mathbf{k}}\right) / K$, which is proportional to the electric field in $y$-direction. Therefore TD's are generated by the external electric field in $y$-direction, forming the "mixed state" of CDW. In order to quantify this point, we study two limiting cases: the dilute and the dense limit of dislocations. 
In the dilute limit, we consider the problem of a single TD: When does the first TD appear as we increase the field strength? The corresponding critical field can be estimated from the difference of the free energy between the single TD state and the TD free state, $\Delta F$. It is given in the limits of $T \ll T_{c}$ and $T \lesssim T_{c}$ as,

$$
\frac{\Delta F}{L_{z}}= \begin{cases}\pi K \gamma W \lambda_{T F}^{-1}-2 \pi e J D_{y}^{e x t} W^{2} & \text { for } T \ll T_{c}, \\ \pi K \gamma \ln \left(W / \xi_{y}\right)-2 \pi e J \lambda_{T F}^{2} D_{y}^{e x t} & \text { for } T \lesssim T_{c} .\end{cases}
$$

In calculating Eq.(66), we assumed that the system is infinite in the $x$-direction and of width $W$ in the $y$-direction, thus introducing an infrared cut-off of $1 / W$ for the wave vector $k_{y}$. Note that " $T \lesssim T_{c}$ " should not include the region too close to $T_{c}$, where the present treatment fails due to the diverging coherence length. In the limit of $T \ll T_{c}$, the critical field is expressed as $D_{c 1}=(\gamma / 4 e) \hbar \omega_{p} \times W^{-1}$, where $\omega_{p}$ is the plasma frequency given by $\sqrt{8 N_{\perp} e^{2} v_{F} / \hbar}$. Therefore, the critical voltage defined by $V_{c 1} \equiv D_{c 1} W$ becomes independent of the sample width. On the other hand, at $T \lesssim T_{c}, D_{c 1}$ is given by $(\gamma / 4 e) \hbar \omega_{p} \times \lambda_{T F}^{-1} \ln \left(W / \lambda_{T F}\right)$. This is larger than the $D_{c 1}$ of the low temperature limit, because the energy gain due to the creation of TD's is reduced by quasi-particles.

In the dense limit, $n_{\mathbf{k}}$ is approximated by a continuous function rather than a function of the positions of individual TD's $\left\{\mathbf{r}_{\mu}\right\}$. This corresponds to neglecting the spatial structure smaller than the spacing between TD's. In this approximation, it is quite simple to evaluate $n_{\mathbf{k}}$ and $\bar{\varphi}_{\mathbf{k}}$ by using Eq. (5). The minimization of the free energy $F_{\text {eff }}$ in terms of $n_{\mathbf{k}}$ gives,

$$
n_{\mathbf{k}}=-\frac{e J}{K} k_{y} \varphi_{\mathbf{k}}
$$

The density of TD's turns out to be proportional to the strength of the electric field in the $y$-direction. On the other hand, the variation of $F_{\text {eff }}$ with respect to $\varphi_{\mathbf{k}}$ gives the Poisson equation,

$$
\left(k^{2}+\Lambda_{\mathbf{k}}^{-2}\right) \bar{\varphi}_{\mathbf{k}}=-4 \pi e\left(J \frac{\gamma^{2}}{k_{\gamma}^{2}} i k_{y} n_{\mathbf{k}}+\rho_{\mathbf{k}}^{e x t}\right) .
$$

Substituting Eq. (7) into Eq. (8), we obtain, $\bar{\varphi}_{\mathbf{k}}=-4 \pi e \rho_{\mathbf{k}}^{e x t} /\left(k^{2}+\Lambda_{m}^{-2}\right)$, where $\Lambda_{m}^{-2}=$ $\lambda_{0}^{-2}+4 \pi e^{2} J^{2} / K$. Note that $\Lambda_{m}$ equals now the Thomas-Fermi screening length of the 
normal state, $\lambda_{T F}$. When we apply the electric field in the $y$-direction, the field penetrates the sample to the depth $\Lambda_{m}$. As we can see from Eq. (]), the TD's are thereby distributed in this surface region where the mixed state is formed.

In case of type-II superconductors (or liquid Helium II), there is another characteristic field, i.e., the upper critical field (or angular velocity), usually denoted as $H_{c 2}\left(\Omega_{c 2}\right)$ [8], which defines a phase transition within the mean field theory. $H_{c 2}\left(\Omega_{c 2}\right)$ can be understood as the field at which the cores of the vortices begin to overlap. In case of the CDW's, when the cores of the TD's begin to overlap, the inter-chain ordering is almost destroyed by strong fluctuations due to the dimensional reduction. We denote this characteristic field as $D_{c 2}$ for convenience, although it is still unclear whether there is a real phase transition or not. This field strength is first realized at the surface where the internal field coincides with the external one. Since the cores begin to overlap when the number density of the TD's exceeds $1 /\left(\xi_{x} \xi_{y}\right)$, the corresponding field strength, $D_{c 2}$, is estimated from Eq. (7) as $D_{c 2}=\pi v_{F} \hbar /\left(e \xi_{x} \xi_{y}\right)$.

In the following, we discuss the experimental aspects of the mixed state with actual CDW materials in mind. We concentrate on the low temperature limit where the effects due to the mixed state are rather clear. Since the TD's modify the screening properties of the system, the mixed state can be observed by measuring the dielectric constant of the system in a capacitor with the transverse electric field. The capacitance should display the crossover from the insulating behavior in the weak field region $\left(V<V_{c 1}\right)$ to the metallic one in the strong field region $\left(V>V_{c 1}\right)$. In the case of $K_{0.3} M_{o O} O_{3}(\gamma=0.01 \sim 0.1$ depending on the direction [1]), $V_{c 1}$ is estimated as $\gamma \times 0.69 \mathrm{~V}$. On the other hand, $D_{c 2}$ is estimated as $\gamma^{-1} \times 2.9 \times 10^{6} \mathrm{~V} / \mathrm{m}$, which can be much larger than $V_{c 1}$ even if we take $W=1 \mu \mathrm{m}$. It should be noted that, in the case of $\mathrm{K}_{0.3} \mathrm{MoO}_{3}$, a simple estimation of the Thomas-Fermi screening length in the normal state, $\lambda_{T F}=\omega_{p} / v_{F}$, gives less than $1 \AA$, which is smaller than the inter-chain spacing $\sim 10 \AA$. This estimation is based on the continuum model of metals and, therefore, we cannot take it too seriously since the continuum approximation is no longer valid at this scale, especially in the transverse direction. However, we expect 
that, in the strong field region, the width of the mixed state can be as narrow as the lattice spacing and most TD's are confined to the outermost layers. In the weaker field region, this width can be much wider.

In the uniform electric field, there can be an additional effect due to the quasi-particles excited through Zener tunneling process [14]. In the WKB approximation [15], the characteristic electric field of Zener breaking is huge in the present configuration $\left(\sim \gamma^{-1} \times 10^{7}\right.$ $\mathrm{V} / \mathrm{m}$ ) due to the narrow band width and the large lattice spacing. Therefore the contributions from the quasi-particle excitations is strongly suppressed and we expect that the mixed state of the condensate can be observed.

The effects of pinning cannot be avoided in realistic CDW systems [16], but are beyond the scope of the present Letter. However, we expect a hysteretic behavior in the observed capacitance. Of course, the magnitude of the hysteresis depends strongly on the inhomogeneity of the sample.

In conclusion, we proposed a new state, i.e., the mixed state, in CDW's, which is realized by applying the transverse electric field. The physical properties are quantitatively discussed based on the Ginzburg-Landau free energy of the CDW and the critical fields which characterize the mixed state are estimated. The mixed state strongly affects the screening properties of the system and is thus detectable through the dielectric constant measurement in a capacitor.

The authors wishes to thank Prof. H. Fukuyama and Prof. H. Matsukawa for stimulating discussions and, Prof. G. Bauer, Dr. Y. Nazarov and Dr. C. Dekker for useful comments. 


\section{REFERENCES}

* Present address: Department of Applied Physics, Delft University of Technology, Lorentzweg 1, 2628 CJ Delft, The Netherlands.

[1] For recent reviews, see G. Grüner,Density Waves in Solids (Addison-Wesley, 1995) and Charge-Density Waves in Solids, ed. L. P. Gor'kov and G. Grüner (Elsevier, Amstredam, 1989).

[2] L. P. Gor'kov, JETP Lett., 38 (1983) 87; JETP, 59 (1985) 1057; I. Batistić, A. Bjeliš and L. P. Gor'kov, J. Phys. France, 45 (1984) 1049.

[3] N. P. Ong, G. Verma and K. Maki, Phys. Rev. Lett. 52 (1984) 663; N. P. Ong and K. Maki, Phys. Rev. B32 (1985) 6582.

[4] S. Ramakurishuna, M. P. Maher, V. Ambegaokar and U. Eckern, Phys. Rev. Lett. 68 (1992) 2066.

[5] J-M. Duan, Phys. Rev. B48 (1993) 4860.

[6] D. Feinberg and J. Friedel, J. Phys. France, 49 (1988) 485

[7] A. Bjeliš, in Applications of Statistical and Field Theory Method to Condensed Matter, ed. D. Baeriswyl et al., NATO ASI Series B: Physics Vol. 218 (Plenum Press, New York, 1990) pp. 325-356.

[8] W. F. Vinen, in Superconductivity, ed. R. D. Parks, (Marcel Dekker, Inc., New York, 1969), pp. 1167-1234.

[9] This idea was first addressed in T. L. Adelman, S. V. Zaitsev-Zotov and R. E. Thorne, Phys. Rev. Lett. 74 (1995) 5264.

[10] M. Hayashi and H. Yoshioka, unpublished.

[11] A. Virosztek and K. Maki, Phys. Rev. B48 (1993) 1368. 
[12] B. Sakita and K. Shizuya, Phys. Rev. B42 (1990) 5586.

[13] N. Nagaosa and M. Oshikawa, J. Phys. Soc. Jpn. 65 (1996) 2241.

[14] P. Monceau, N. P. Ong, A. M. Portis, A. Meerschaut and J. Rouxel, Phys. Rev. Lett. 37 (1976) 602.

[15] The characteristic field of Zener breaking is given by $\left(\pi^{2} / 4\right) \Delta^{2} /(D e a)$ where $\Delta, D$ and $a$ are energy gap, band width and lattice spacing, respectively. See J. M. Ziman, Principles of the Theory of Solids (Cambridge Univ. Press, Cambridge, England, 1972), 2nd ed., Sect. 6.8.

[16] H. Fukuyama and P. A. Lee, Phys. Rev. 17 (1978) 535; P. A. Lee and T. M. Rice, Phys. Rev. 19 (1979) 3970. 